\title{
HOMOSSEXUALIDADE E ENVELHECIMENTO E AS POSSÍVEIS
} VULNERABILIDADES

Luciana de Almeida da Cunha - Enfermeira, Doutoranda Pelo Instituto de Geriatria e Gerontologia-PUCRS, Bolsista CAPES/CNPq. E-mail: luciana.dealmeida@Hotmail.com

José Roberto Goldim - Programa de Pós-Graduação em Ciências Médicas, Universidade Federal do Rio Grande do Sul, Brasil. E-mail: jrgoldim@gmail.com

\section{RESUMO}

Introdução: Vulnerabilidade é um construto multidimensional que se refere a um contexto dinâmico em que há risco de desenvolver problemas de saúde, seja por uma inadequação econômica, social, psicológica, familiar, cognitiva ou de recursos físicos. Deve-se considerar diversos aspectos da interação biopsicossocial como, por exemplo, as relações sociais e também de trabalho. Objetivo: Verificar a associação de relatos de vulnerabilidades em pessoas idosas e homossexuais em artigos publicados na área da saúde. Método: Levantamento bibliográfico através de três bases de dados: Pubmed, Scopus e Web of Science. Critérios de inclusão: artigos relacionados a questões de envelhecimento e homossexualidade, publicados nos últimos cinco anos. Critério de exclusão: os artigos que não puderam ser acessados na íntegra. Os textos foram avaliados de forma qualitativa visando estabelecer categorias de análise vinculadas à vulnerabilidade. Resultados: Foram analisados 279 artigos dos 349 artigos localizados. Em 252 artigos, a vulnerabilidade física foi a predominante. Em outros 22 artigos foi possível destacar a vulnerabilidade social, representada pelas situações de ter que viver na invisibilidade acerca da própria orientação sexual, por medo do preconceito ou do rechaço. Finalmente, em cinco artigos houve a menção de que a variável sexualidade não era abordada no atendimento de pacientes idosos. Conclusão: A vulnerabilidade estática é aquela associada ao simples fato de ser velho e homossexual, enquanto que a vulnerabilidade dinâmica é a decorrente de situações que envolvam as relações sociais associadas a estas características. Os resultados evidenciam a necessidade de incluir este tema como objeto de reflexão por parte da sociedade.

Palavras chave: Envelhecimento; vulnerabilidade; minoria social.

Agradecimento: Ao apoio da Coordenação de Aperfeiçoamento de Pessoal de Nível Superior - Brasil (CAPES) - Código de Financiamento 001. 